\title{
Juges constitutionnels et parlements : conflits de legitimite ou nouvelle organisation des pouvoirs? Le cas de la suisse
}

\section{Los Jueces constitucionales y parlamentos : conflictos de legitimidad o nueva organización de poderes? El caso de Suiza}

Michell Hottelier ${ }^{1}$

\section{INTRODUCTION}

Comme le relève le document introductif de la XXVII ${ }^{\text {ème }}$ Table ronde internationale de justice constitutionnelle, la thématique "Juges constitutionnels et Parlements " représente assurément un sujet classique. L'opposition traditionnelle, inhérente a ce thème, entre souveraineté et Etat de droit, entre politique et judiciaire, entre démocratie et respect du droit, est présente en Suisse aussi. Elle y prend toutefois une tournure un peu particulière, en raison de la complexité du système institutionnel propre à ce pays.

Le système institutionnel helvétique voit en effet coexister souveraineté parlementaire, démocratie directe et une forme de juridiction constitutionnelle agencée de façon très différente selon qu'elle s'exerce à l'égard d'actes normatifs de rang fédéral ou cantonal. La démocratie directe comme le fédéralisme sont par conséquent deux éléments centraux avec lesquels il faut compter dans l'abord de cette problématique, raison pour laquelle le présent rapport sera fréquemment amené à traiter séparément les questions posées sous l'angle du droit fédéral ou cantonal.

\section{LE CADRE INSTITUTIONNEL DES RELATIONS ENTRE LES PARLEMENTS ET LES JUGES CONSTITUTIONNELS}

\subsection{L'organisation des relations institutionnelles entre parlement et juridiction constitutionnelle}

\subsubsection{La forme de l'Etat}

La structure fédérale sur laquelle la Suisse repose depuis 1848 exerce une influence profonde sur la structure de ses institutions. L'agencement des fonctions parlementaire et judiciaire, y compris de la juridiction constitutionnelle, ne fait pas exception.

À l'échelon national, c'est l'Assemblée fédérale qui exerce la fonction parlementaire. La structure est calquée, d'un point de vue historique, sur le modèle institutionnel des Etats-Unis : deux Chambres, dont l'une (Conseil national, 200 députés) représente le

\footnotetext{
${ }^{1}$ Professeur à l'Université de Genève. Profesor de la Universidad de Ginebra.
} 
peuple; l'autre (Conseil des Etats, 46 membres) représente les cantons. Dans chaque canton, l'activité parlementaire est exercée par un organe ad hoc, généralement appelé Grand Conseil dans la partie francophone du pays, qui est élu au suffrage universel.

La Suisse connaît, à l'image également des Etats-Unis, un système diffus de juridiction constitutionnelle. II n'y a donc pas, en Suisse, de Cour constitutionnelle détenant l'exclusivité ou le monopole de l'interprétation et de l'application de la législation constitutionnelle, fédérale ou cantonale. Chaque instance est ainsi habilitée, à son niveau et selon son degré de compétence, à exercer le contrôle de la constitutionnalité, le Tribunal fédéral assumant la fonction de juridiction suprême pour l'interprétation du droit constitutionnel aussi bien fédéral que cantonal.

Des organes politiques sont aussi chargés d'exercer le contrôle visant au respect de la Constitution, à l'image de l'Assemblée fédérale lorsqu'elle contrôle la validité d'une initiative populaire ou lorsqu'elle accorde sa garantie à une constitution cantonale. Un parlement cantonal exerce la même fonction lorsqu'il statue sur la validité d'une initiative populaire locale, de rang constitutionnel ou législatif. En d'autres termes, contrairement à la conception dominante au sein d'autres ordres juridiques, la juridiction constitutionnelle désigne en Suisse une fonction plutôt qu'un organe ${ }^{2}$.

L'agencement des rapports entre les parlements et les organes habilités à exercer la juridiction constitutionnelle est assez complexe, car il repose sur une structure croisée : on peut ainsi distinguer les rapports entre le Parlement fédéral et le Tribunal fédéral, d'une part, des rapports entre parlements cantonaux et autorités judiciaires cantonales, d'autre part.

Une remarque doit être faite s'agissant du statut de l'Assemblée fédérale. À teneur de l'article 148 alinéa 1 de la Constitution fédérale du 18 avril 1999, en vigueur depuis le $1^{\text {er }}$ janvier 2000 (recueil systématique du droit fédéral suisse), cet organe exerce " l'autorité suprême de la Confédération, sous réserve des droits du peuple et des cantons ». Pour notre propos, deux éléments découlent de cette disposition ${ }^{3}$.

Le premier tient au fait que, dans l'agencement institutionnel fédéral, le Parlement national dispose d'une sorte de primauté, soit d'une situation juridiquement supérieure à celle des autres autorités, comme le soulignent les Professeurs Aubert et Mahon. Cette primauté tient au mode de désignation de l'Assemblée fédérale, dont les deux Chambres sont élues directement, par le corps électoral fédéral pour ce qui du Conseil national et par celui des cantons, à leur niveau propre, pour ce qui est du Conseil des Etats. Les autres pouvoirs de rang fédéral sont pour leur part au bénéfice d'une légitimité distincte, dérivée. Tant le Conseil fédéral que les juges fédéraux sont en effet élus par l'Assemblée fédérale. Le Gouvernement suisse n'a jamais été élu au suffrage populaire direct, cette question revenant régulièrement on pourrait parler de débat récurrent - sur la scène politique fédérale ${ }^{4}$.

\footnotetext{
2 Voir les développements de Maya Hertig Randall, L'internationalisation de la juridiction constitutionnelle : défis et perspectives, Revue de droit suisse 2010 II, p. 230.

${ }^{3}$ Pour plus de détails, voir en particulier Jean-François Aubert/Pascal Mahon, Petit commentaire de la Constitution fédérale de la Confédération suisse du 18 avril 1999, Zurich 2003, p. 1156.

${ }^{4}$ Sur l'aspect historique, voir Jean-François Aubert, Traité de droit constitutionnel suisse, Neuchâtel 1967, p. 73 et 531 .
} 
Le second élément tient à la réserve des "droits du peuple et des cantons 》 qu'énonce l'article 148 alinéa 1 Cst. Ce membre de la disposition signifie que la Constitution fédérale, pour être issue du vote obligatoire du peuple et des cantons, prime sur l'Assemblée fédérale et donc sur les actes que celle-ci adopte. Le Parlement doit, en d'autres termes, respecter la Constitution formelle dans l'exercice des activités que cette dernière lui attribue. Le problème étant, comme nous le voir, que le contrôle institutionnel sur le sujet est largement incomplet.

Ce tempérament à la primauté de l'Assemblée fédérale, qui soumet celle-ci aux décisions du constituant, est en effet largement atténué par la règle de l'article 190 Cst. Cette disposition fait en effet obligation au Tribunal fédéral et, à son exemple, aux autres autorités d'appliquer les lois fédérales ${ }^{5}$.

Les lois fédérales sont en effet votées par l'autorité suprême de la Confédération et passibles, de surcroît, du référendum facultatif, si la demande émane de 50'000 citoyens ou de huit cantons dans les 100 jours suivant leur adoption (art. 141 Cst.). Autrement dit, le vote démocratique des représentants du peuple et des cantons, allié pour la circonstance au suffrage populaire direct, fait obstacle au contrôle judiciaire de constitutionnalité. L'adage «Vox populi, vox dei » résume, pour la circonstance, bien la situation. Face au juge, c'est donc l'acte parlementaire qui prime, fût-ce au détriment du respect de la Constitution et, en particulier, des droits fondamentaux qu'elle consacre. L'opposition classique entre souveraineté, en l'occurrence parlementaire et Etat de droit, entre politique et juridique, est ici flagrante.

L'agencement du système juridictionnel suisse réserve de larges compétences aux cantons. Le fédéralisme judiciaire est, en ce domaine, très prononcé en Suisse, puisque ce sont le plus souvent les juges des cantons qui sont, dans l'ordre du contentieux, les premiers acteurs qui sont appelés à rendre la justice. «On peut sans doute voir dans le système de justice « cantonalisée » une particularité du fédéralisme suisse, un fédéralisme judiciaire très prononcé, qui le distingue d'autres systèmes, tel le régime américain, dans lequel se côtoient et se superposent deux organisations judiciaires complètes, celle de chacun des Etats et celle de I'Union" (Aubert, Mahon, 2003).

Dans certains cantons, une forme de juridiction constitutionnelle abstraite est possible à l'égard des normes locales. Ce type de contrôle peut être complet ou ne porter, au contraire, que sur certains actes. S'il est complet, il concerne les lois et les règlements locaux. S'il est en revanche retreint, il ne vise pas les lois cantonales, ce qui est aussi une manière de réserver la part belle à la primauté du débat parlementaire. Le contrôle judiciaire abstrait de constitutionnalité est toutefois rare et n'existe que dans un nombre limité de cantons comme le Jura ou, plus récemment, le canton de Vaud ${ }^{6}$. La grande majorité d'entre eux ne permet pas, en effet, à un cénacle de juristes d'examiner abstraitement la conformité des lois votées par le parlement cantonal ou d'autres actes normatifs à la Constitution fédérale ou même

\footnotetext{
${ }^{5}$ Voir Hertig Randall (note 1), p. 237ss. et les nombreuses références citées.

${ }^{6}$ Voir Andreas Auer/Giorgio Malinverni/Michel Hottelier, Droit constitutionnel suisse, vol. I, L'Etat, $2^{\text {ème }}$ éd., Berne 2006, p. 797.
} 
cantonale. L'argument du contrôle politique et démocratique est ainsi également largement présent pour expliquer l'absence de judicial review de type abstrait sur le terrain local.

Il en va différemment en ce qui concerne le contrôle concret de constitutionnalité. Tous les cantons sont en effet obligés de permettre au corps judiciaire de vérifier la conformité des actes normatifs cantonaux à la Constitution fédérale. De même, les cantons ont l'obligation d'écarter l'application du droit cantonal contraire au droit supérieur, dans son ensemble. Ici, l'argument démocratique cède le pas face aux exigences du fédéralisme : aucun acte normatif local ne saurait, au nom de la loi d'autonomie propre à la structure fédéraliste, faire obstacle à la correcte application et au respect du droit fédéral, y compris bien entendu à la Constitution fédérale. Cette exigence résulte du principe de la primauté du droit fédéral tel qu'il découle de l'article 49 alinéa 1 Cst (Auer, Malinverni G, Hottelier M 2006). La juridiction constitutionnelle vient ici au secours du fédéralisme, au nom de la nécessité de veiller à une application uniforme du droit fédéral dans tous les cantons. Le Tribunal fédéral le rappelle de temps à autre ${ }^{7}$.

Un arrêt rendu le 8 octobre 1986 a par exemple permis à la Haute Cour de connaître, sur contrôle abstrait, d'un recours dirigé contre une disposition de la nouvelle loi genevoise de procédure administrative ${ }^{8}$. Celle-ci excluait de tout contrôle incident de constitutionnalité les lois votées par le Grand Conseil genevois ou acceptées en référendum par le peuple. En l'occurrence, les juges fédéraux ont admis le recours et relevé l'inconstitutionnalité de la disposition en cause dans la mesure où celle-ci faisait obstacle au contrôle préjudiciel de la conformité des lois cantonales au droit fédéral. Pour la question du contrôle de conformité de la législation cantonale à la Constitution du canton, le Tribunal fédéral a laissé la question ouverte ${ }^{9}$. II a toutefois annulé la norme litigieuse dans son ensemble, de manière à permettre au législateur cantonal de rédiger une nouvelle disposition.

\footnotetext{
${ }^{7}$ Voir par exemple ATF 127 I 185, 187s. R. : « Nach Lehre und Rechtsprechung sind die kantonalen Gerichte nicht nur berechtigt sondern verpflichtet, auf Verlangen eines Rechtsuchenden das anzuwendende kantonale Recht vorfrageweise auf seine Übereinstimmung mit der Bundesverfassung zu prüfen. Damit verbunden ist grundsätzlich auch die Pflicht, als verfassungswidrig erkanntes Recht im Einzelfall nicht anzuwenden ». Voir également ATF 117 Ia 262 Y. u. Mitb. ; 112 Ia 311 X. ; 106 Ia 383 Gemeinde Zuoz.

${ }^{8}$ Arrêt du Tribunal fédéral en la cause L. A. c. Grand Conseil de la République et canton de Genève, Revue de droit administratif et de droit fiscal 1987, p. 433 et les autres références doctrinales et jurisprudentielles citées.

${ }_{9}$ Arrêt du Tribunal fédéral en la cause L.A. c. Grand Conseil de la République et canton de Genève, Revue de droit administratif et de droit fiscal 1987, p. 436 : «Il faut donc s'en tenir sur ce point au principe selon lequel les cantons peuvent organiser leur procédure comme ils l'entendent et qu'ils sont donc libres d'introduire le contrôle de la conformité de leurs lois avec la constitution cantonale ou de l'interdire, même à titre préjudiciel, en réservant cet examen, sur le plan cantonal, au seul législateur. La différence qui peut en résulter par rapport au contrôle préjudiciel de la conformité des lois cantonales avec le droit fédéral n'est pas choquante, car elle découle justement du système fédératif et de l'article 3 Cst. »
} 


\subsubsection{La structure du Parlement}

Par rapport à d'autres Etats, fédéraux ou non, la structure bicamérale du Parlement suisse est parfaite au sens technique du terme, c'est-à-dire égalitaire : les deux Chambres disposent en effet des mêmes attributions, sans prédominance possible des voix exprimées au sein de l'une par rapport à l'autre.

L'article 148 alinéa 2 in fine Cst., dont le titre marginal mentionne d'ailleurs la structure bicamérale de l'Assemblée fédérale, exprime cette règle de manière claire, en disposant que le Conseil national et le Conseil des Etats sont dotés " des mêmes compétences". L'article 156 alinéa 2 Cst. précise aussi que "les décisions de l'Assemblée fédérale requièrent l'approbation des deux conseils ".

Le bicamérisme égalitaire pratiqué en Suisse a pour conséquence que toute loi fédérale doit impérativement recueillir une majorité des voix au sein de chacune des deux Chambres. II s'ensuit que le processus parlementaire est long et presque toujours incertain, face à la menace permanente que l'éventualité d'un référendum fait, à la manière d'une épée de Damoclès, peser sur les travaux législatifs de l'Assemblée fédérale.

Le caractère égalitaire du bicamérisme helvétique déploie aussi une conséquence plus inattendue, mais néanmoins logique lorsque le juge, le plaideur ou l'auteur de doctrine a à se pencher sur le texte d'une loi fédérale pour en interpréter le sens. La recherche du sens réel de la norme, que ce soit selon la méthode d'interprétation littérale, historique, logique ou même téléologique pousse bien souvent l'exégète à aller rechercher les éléments idoines dans les travaux qui se sont déroulés dans chacun des deux Conseils, en s'interrogeant de surcroît sur la signification éventuellement différente que la version allemande, française et italienne - dont chacune fait également foi - de la norme peut impliquer ${ }^{10}$.

Les deux Chambres sont par ailleurs composées différemment, le Conseil national étant élu à la proportionnelle (art. 149 al. 2 Cst.), alors que le Conseil des Etats est le plus souvent élu à la majoritaire à deux tours ${ }^{11}$. Elles siègent enfin séparément (art. 156 al. 1 Cst.) au sein du Palais fédéral situé à Berne et leurs membres ne sont soumis à aucune instruction.

Le seul cas dans lequel une Chambre peut avoir une sorte de prépondérance par rapport à l'autre est l'hypothèse dans laquelle les deux Conseils siègent ensemble. L'article 157 alinéa 1 Cst. prévoit ce cas de figure, qui est toutefois limité à trois situations précises: l'élection d'organes comme le Conseil fédéral ou les juges fédéraux, les conflits de compétences entre les autorités fédérales suprêmes - cas

\footnotetext{
${ }^{10}$ Voir par exemple ATF 136 II 415 A. und Mitb., au sujet du caractère exhaustif de la réglementation fédérale sur l'assistance au suicide; ATF 130 V 479 Assura, au sujet de la participation des cantons au financement des coûts d'hospitalisation de leurs habitants dans le cadre de la loi fédérale sur l'assurance maladie obligatoire; ATF 129 I 402 Schweizerische Vereinigung für Straflosigkeit des Schwangerschaftsabbruchs und Mitb., au sujet de l'interprétation de la norme pénale relative à l'interruption volontaire de grossesse.

${ }^{11}$ Les cantons édictent les règles applicables à l'élection de leurs députés au Conseil des Etats, conformément à l'article 150 alinéa 3 Cst. Dans le canton du Jura et, plus récemment dans celui de Neuchâtel, les conseillers aux Etats sont élus à la proportionnelle.
} 
plutôt rare - ou les recours en grâce. En pareille circonstance, les membres du Conseil des Etats, moins nombreux, sont nécessairement majorisés, à plus de quatre contre 1, par les voix de leurs collègues du Conseil national (Aubert, Mahon, 1999).

La structure du Parlement fédéral a incontestablement été conçue pour contribuer à asseoir la légitimité institutionnelle et politique des lois qu'adopte l'Assemblée fédérale au sens de l'article 164 Cst. II s'agit de l'une des expressions les plus tangibles de la suprématie parlementaire qu'énonce l'article 148 alinéa 1 Cst., précédemment évoqué. Toute loi fédérale procède ainsi de la volonté, majoritairement et doublement exprimée, des représentants du peuple et des cantons. Et comme ces lois sont sujettes à consultation populaire directe par la voie du référendum, la logique selon laquelle le contrôle démocratique prime les attributions réservées à la juridiction constitutionnelle est assurée.

Cette logique historique d'exclusive apparaît toutefois, à notre sens du moins, dépassée de nos jours, en raison notamment du développement de l'Etat de droit et, corrélativement, de l'importance de plus en plus marquée que les droits fondamentaux ont gagnée au cours des âges. L'Etat, aujourd'hui, n'est pas que démocratique. II l'est toujours, bien entendu, mais plus seulement. II est aussi devenu un Etat de droit, libéral et social, sans rapport hiérarchique entre ces diverses composantes. C'est pourquoi la possibilité de permettre un contrôle judiciaire de la conformité des lois fédérales à la Constitution est aujourd'hui de plus en plus souvent évoquée. En Suisse, cette réforme, on l'aura compris, passe toutefois par une révision formelle de la Constitution fédérale.

Au sein des cantons, la structure du parlement est monocamérale. II n'y a donc pas, dans aucun canton, de chambre du peuple et de chambre des communes. Le bicamérisme est inconnu, partout, au niveau cantonal (Auer et al. 2006).

Les lois cantonales sont soumises au référendum obligatoire ou facultatif, en fonction des types de normes et des cantons. Dans le canton de Genève par exemple, les lois votées par le Grand Conseil sont le plus souvent soumises au référendum facultatif. Le référendum doit être demandé dans un délai de quarante jours par sept mille citoyens. Celles qui touchent au domaine fiscal d'une part ou à la protection des locataires sont en revanche passibles du référendum obligatoire ${ }^{12}$.

La structure institutionnelle des parlements cantonaux rappelle, à sa manière, celle de l'Assemblée fédérale: très démocratique dans la composition de l'organe parlementaire, qui est partout élu par le peuple, elle ouvre en outre largement la voie au contrôle référendaire. C'est pourquoi le contrôle juridictionnel abstrait de la conformité des lois cantonales à la constitution locale est très peu répandu.

\footnotetext{
${ }^{12}$ Le problème pouvant naturellement se poser de déterminer à quel type de référendum, obligatoire ou facultatif, sont soumises les lois qui contiennent l'une ou l'autre disposition touchant le droit fiscal ou la protection des locataires. Pour un cas de ce genre, voir l'arrêt du Tribunal fédéral du 9 novembre 2010 dans la cause Alberto Velasco et consorts c. Conseil d'Etat du canton de Genève, Pratique juridique actuelle 2011, p. 263
} 


\subsection{Les systèmes d'influence réciproque existants entre les parlements et les cours constitutionnelles}

\subsubsection{L'apport des parlements au processus de nomination des membres des cours constitutionnelles}

Il existe en Suisse trois tribunaux fédéraux : le Tribunal pénal fédéral, qui siège à Bellinzone, le Tribunal administratif fédéral, qui siège à Berne et le Tribunal fédéral, dont le siège se trouve à Lausanne, mais dont les Chambres de droit social délibèrent à Lucerne. Alors que le Tribunal pénal fédéral, le Tribunal administratif fédéral ou d'autres instances fédérales comme les commissions d'estimation en matière d'expropriation ou les tribunaux militaires disposent de compétences limitées à certains domaines du droit, le Tribunal fédéral est doté d'une compétence générale.

Conformément à l'article 188 alinéa 1 Cst. le Tribunal fédéral est l'autorité judiciaire suprême de la Confédération. Face à l'autorité législative et exécutive fédérale, le Tribunal fédéral forme dans cette mesure le troisième pouvoir de la Confédération (Aubert, Mahon, 1999). En règle générale, il ne statue qu'après les instances cantonales ou fédérales.

La juridiction constitutionnelle qu'exerce le Tribunal fédéral n'intervient que dans un cadre contentieux, c'est-à-dire sur recours. Le contrôle judiciaire préventif des lois, antérieur à leur promulgation, ou même consultatif, n'existe pas sur le plan fédéral. L'article 189 alinéa 1 Cst. exprime clairement cette règle, en soulignant que le Tribunal fédéral connaît de "contestations ", ce qui suppose toujours l'existence d'un litige opposant deux ou plusieurs parties. L'article 191b alinéa 1 Cst. pose également l'exigence d'une contestation pour la saisine des autorités judiciaires cantonales dans les domaines relevant du droit civil, pénal ou public.

L'apport du Parlement fédéral au processus de nomination des membres du Tribunal fédéral est central et déterminant. Les juges fédéraux, dont le nombre, prévu par la loi, oscille entre trente-cinq et quarante-cinq, leurs suppléants, ainsi que le président et vice-président du Tribunal fédéral, sont en effet élus par l'Assemblée fédérale, laquelle siège, pour la circonstance, en conseils réunis, conformément aux articles 157 alinéa 1 let. a et 168 alinéa 1 Cst. La durée du mandat de juge au Tribunal fédéral est de six ans, renouvelable jusqu'à l'âge de soixante-huit ans. La solution suisse frappe, dans une perspective comparative, par la brièveté de la durée du mandat des juges fédéraux, de même que par la possibilité d'une reconduction et donc d'un réexamen régulier des candidatures ${ }^{13}$.

Tout citoyen suisse bénéficiant des droits politiques est éligible au Tribunal fédéral (art. 143 Cst.). L’Assemblée fédérale choisit les juges fédéraux de telle manière que les langues officielles de la Confédération, soit l'allemand, le français et l'italien (art. 70 al. 1 Cst.) soient représentées. Cette règle de choix, antérieurement prévue par la Constitution fédérale, a été abrogée, mais toutefois maintenue dans la pratique, pour d'évidentes raisons. Lors de l'élection des juges au Tribunal fédéral, l'Assemblée

\footnotetext{
${ }^{13}$ Sur le sujet, voir les développements de Hertig Randall (note 1), p. 335 ; voir également Regina Kiener, Richterliche Unabhängigkeit, Berne 2001, p. 257s.
} 
fédérale accorde par ailleurs une représentation équitable aux deux confessions chrétiennes, de même qu'aux principaux partis politiques représentés à l'échelon national. En règle générale, les candidats sont d'ailleurs présentés par un parti politique (Aubert, Mahon, 1999).

Les postes de juge fédéral son publiquement mis au concours et paraissent ainsi dans la presse. Suite au dépôt des candidatures, la Commission judiciaire de l'Assemblée fédérale examine les dossiers. La Commission judiciaire est composée de douze membres du Conseil national et de cinq du Conseil des Etats, chaque groupe politique représenté aux Chambres ayant droit à un siège au sein de la commission. Elle soumet ensuite au plenum de l'Assemblée fédérale ses propositions pour l'élection, conformément à l'article 40a de la loi sur l'Assemblée fédérale, du 13 décembre $2002^{14}$. L'élection proprement dite se déroule au scrutin majoritaire (art. 137 LParl.).

Si l'apport de l'Assemblée fédérale est déterminant dans la composition du Tribunal fédéral, aucune espèce de contrôle n'est bien entendu pratiqué, en vertu du principe de la séparation des pouvoirs, sur l'activité des juges au quotidien. Durant l'exercice de leur mandat, ces derniers ne peuvent être membres de l'Assemblée fédérale ou du Conseil fédéral, ni exercer aucune autre fonction au service de la Confédération.

L'article 191c Cst. dispose que "dans l'exercice de leurs compétences juridictionnelles, les autorités judiciaires sont indépendantes et ne sont soumises qu'à la loi ». Ses arrêts ne peuvent être annulés ou modifiés que par lui-même et conformément aux dispositions de la loi sur le Tribunal fédéral (art. 2 al. 2) ${ }^{15}$. Cela étant, l'Assemblée fédérale exerce la haute surveillance sur le Tribunal fédéral. C'est également elle qui approuve chaque année le budget, les comptes et le rapport de gestion du Tribunal fédéral, conformément à l'article 3 LTF.

Le processus de sélection et de nomination des juges sur le plan cantonal ressemble à celui qui est pratiqué sur le plan fédéral. C'est très souvent le parlement qui élit les juges, dont les candidatures sont présentées par les formations politiques. Parfois, l'élection revient au corps électoral. A Genève par exemple, les juges sont en principe élus par le peuple au scrutin majoritaire pour une durée de six ans, conformément à l'article 132 de la Constitution cantonale. Toutefois, l'élection est tacite sur la base des propositions faites par le Grand Conseil lorsque le nombre de candidats est identique au nombre de sièges à pourvoir.

2.1.2. Le rôle du Parlement sur la mise en place et les modalités de fonctionnement de la juridiction constitutionnelle

Les modalités entourant l'institution et le fonctionnement de la juridiction constitutionnelle se répartissent en Suisse entre trois sources, distinctes, mais complémentaires : la Constitution fédérale, la législation fédérale et la jurisprudence du Tribunal fédéral.

\footnotetext{
${ }^{14}$ Ci-après : LParl. ; RS 171.10.

${ }^{15}$ Ci-après : LTF ; RS173.110.
} 
La Constitution fédérale comprend un chapitre bref, mais néanmoins assez complet, consacré au Tribunal fédéral. Concrétisant le principe de la séparation des pouvoirs, les articles 188 et suivants Cst. énoncent les règles de base à cet effet, en instituant le Tribunal fédéral et en définissant ses compétences: «le Tribunal fédéral est ainsi présenté comme l'autorité judiciaire suprême de la Confédération (art. 188 al. 1 Cst.) et son indépendance, de même que celle des autres autorités judiciaires du pays est consacrée (art. 191c Cst.) ». "L'indépendance de la justice apparaît comme le pendant institutionnel de l'article 30 Cst., qui fonde le droit du justiciable à un tribunal indépendant et impartial » (Poltier, E., 2011).

Les compétences du Tribunal fédéral sont énumérées à l'article 189 Cst. La juridiction constitutionnelle n'y est pas, de manière générale, citée ès qualité. Le constituant a en effet opté pour une liste de motifs ou de griefs ouvrant la voie à la saisine de la Cour suprême plutôt que pour l'énumération du type de contrôle pratiqué. La juridiction constitutionnelle prend toutefois place, de manière générale, parmi les contestations pour violation du droit fédéral qu'énonce l'article 189 alinéa 1 lettre a Cst. Le contentieux mettant en cause la Constitution fédérale fait naturellement partie de ce chef de compétence (Aubert, Mahon, 1999). La juridiction constitutionnelle est ainsi intégrée à la juridiction ordinaire du Tribunal fédéral.

Les contestations fondées sur la violation des droits constitutionnels cantonaux, par quoi l'on entend les droits fondamentaux garantis par les constitutions cantonales, sont elles aussi expressément mentionnées comme relevant de la compétence du Tribunal fédéral (art. 189 al. 1 let. d Cst.). Cette mention ne s'explique pas seulement, d'un point de vue historique, par l'importance que revêtent, en matière de protection des droits individuels, les constitutions des vingt-six cantons. Elle trouve aussi, d'un point de vue juridique, une raison d'être dans la structure fédérale du pays, qui a pour conséquence que l'application du droit cantonal, pour procéder d'ordres juridiques distincts du droit fédéral, échappe en principe à la connaissance des juges fédéraux. Sauf exception, ces derniers n'ont donc en principe pas voix au chapitre en ce domaine (Aubert, Mahon, 1999). Lorsque c'est le cas, la Constitution et la législation fédérales doivent le dire.

La Constitution fédérale énonce de manière explicite une autre compétence relevant de la juridiction constitutionnelle : le contentieux en matière de droits politiques, qu'il soit de rang fédéral ou cantonal, conformément à l'article 189 alinéa 1 lettre f Cst.

De même, l'article 189 alinéa 2 Cst. précise que les différends entre la Confédération et les cantons ou entre les cantons eux-mêmes est soumis à la compétence des juges fédéraux. Ce type de litige, qui porte sur la répartition des compétences entre autorités fédérales et cantonales, représente une autre dimension importante de la juridiction constitutionnelle, outre le respect des droits fondamentaux et le contentieux des droits politiques.

L'exclusion du contrôle abstrait de constitutionnalité à l'égard des actes normatifs votés par l'Assemblée fédérale et le Conseil fédéral est prévue à l'article 189 alinéa 4 Cst. À teneur de cette disposition, les actes de l'Assemblée fédérale et du Conseil fédéral ne peuvent pas être portés devant le Tribunal fédéral. Lorsqu'il est possible, le contrôle de la constitutionnalité des actes fédéraux n'est ainsi que concret concret (Aubert, Mahon, 1999). 
La Constitution énonce, à son article 190, une autre règle dont l'impact est déterminant dans le fonctionnement de la juridiction constitutionnelle. En proclamant que le Tribunal fédéral et, à son exemple, les autres autorités sont tenus d'appliquer les lois fédérales et le droit international, la disposition ne rappelle pas seulement, de manière positive, que le rôle des juges en Suisse est de veiller au contrôle de la légalité, d'une part, et de la conventionnalité, d'autre part. La règle doit plutôt s'entendre par la négative: elle signifie que nulle autorité, administrative ou judiciaire, n'est légitimée à refuser d'appliquer une loi votée par l'Assemblée fédérale au motif que celle-là contrevient à la Constitution.

Cette conception très statique du juge et de son rôle, réduit historiquement au rôle de "bouche qui prononce les paroles de la loi » («ius-dicere»), traduit la pensée dominante en Suisse et, plus largement, en Europe, au $19^{\text {ème }}$ siècle, au nom d'une vision figée de la séparation des pouvoirs, qui sépare de manière étanche le phénomène (politique, donc démocratique) de création du droit de celui (juridique, donc technique) de l'application des normes ${ }^{16}$.

L'interprétation littérale de l'article 190 Cst. laisse toutefois indécise une question centrale: celle de l'hypothèse d'une loi fédérale elle-même contraire au droit international. Quel type de contrôle doit être pratiqué en pareille hypothèse : le contrôle de la légalité ou celui de la conventionnalité ? Quel acte prime : la loi fédérale ou le droit international en cas de conflit irréductible entre ces deux sources du droit ? Cette question a été tranchée par la jurisprudence, comme nous le verrons plus loin. Pour le surplus, la mise en place des règles posées par la Constitution fédérale revient à l'Assemblée fédérale, conformément aux articles 164 alinéa 1 lettre f et 188 alinéa 2 Cst.

L'exercice proprement dit et les modalités entourant le contrôle judiciaire sont réglés par la loi sur le Tribunal fédéral. Adoptée le 17 juin 2005 et entrée en vigueur le $1^{\text {er }}$ janvier 2007, cette loi énonce le régime général de l'organisation, des compétences et de la procédure qui sont applicables à la Cour suprême ${ }^{17}$. La loi prévoit, en particulier, un régime des voies de recours ordonné de manière assez logique, en permettant en principe au plaideur d'invoquer dans une même écriture des moyens ressortissant au contrôle de la légalité, de la constitutionalité et de la conventionnalité.

Le même recours permet donc de déclencher, voire cumuler lorsque cela est possible, les trois types de contrôle, rangeant la juridiction constitutionnelle au nombre des compétences ressortissant à la juridiction ordinaire. De même, la loi sur le Tribunal fédéral précise de quelle manière le contentieux portant sur la répartition des compétences entre la Confédération et les cantons au sens de l'article 189 alinéa 2 Cst. s'exerce.

Le rôle qu'assume le législateur dans l'agencement du mode saisine de la Cour suprême est donc central. Central, mais nullement exclusif : comme les lois fédérales

\footnotetext{
${ }^{16}$ Hertig Randall (note 1), p. 238s. et les références citées, qui relève en particulier que ce n'est qu'au cours du $20^{\text {ème }}$ siècle que la juridiction constitutionnelle conquit, en plusieurs vagues, le continent européen, plaçant alors la Suisse et d'autres Etats attachés à la primauté du parlement dans une position minoritaire.

${ }^{17}$ Au sujet de cette loi, voir AIJC XXI-2005, p. 717.
} 
sont passibles du référendum populaire, le contrôle populaire des lois votées par le Parlement fédéral n'est jamais exclu d'emblée. C'est du moins ce qui s'est passé à la fin des années 1980. L'Assemblée fédérale avait, à l'époque, mis au point une réforme de la loi fédérale d'organisation judiciaire qui tendait à limiter, notamment, la saisine du Tribunal fédéral en fixant, pour certaines catégories de litiges, des valeurs litigieuses minimales. Soumise au référendum, cette loi fut finalement refusée par le peuple le $1^{\text {er }}$ avril 1990.

Enfin, last but not least, le fonctionnement du Tribunal fédéral et la manière d'exercer les compétences qui reviennent à cette instance sont largement tributaires des règles dégagées par la jurisprudence. Dans le domaine de la juridiction constitutionnelle, c'est le Tribunal fédéral qui a par exemple développé la méthode de l'interprétation conforme de lois à la Constitution en précisant qu'il lui appartient, en cas de plusieurs interprétations possibles d'une loi, singulièrement d'une loi fédérale, d'opter pour celle qui respecte la Constitution fédérale.

Depuis une jurisprudence inaugurée en $1969^{18}$, le Tribunal fédéral admet en effet que, lorsqu'il se trouve confronté plusieurs interprétations d'une loi fédérale, il doit opter pour celle qui est de nature à conduire au respect de la Constitution. L'approche lui permet alors d'accorder la préférence à l'interprétation qui fasse apparaître la loi comme compatible avec la Constitution. Pour arriver à cette fin, le Tribunal fédéral est tenu d'examiner le rapport qu'entretient la loi avec la Constitution, en sorte que c'est bien une facette de la juridiction constitutionnelle qui est ainsi exercée (Auer et al, 2006).

De même, dans le cadre du contrôle abstrait de constitutionnalité des actes normatifs cantonaux, l'interprétation conforme permet aux juges fédéraux de préciser la portée des normes cantonales en leur conférant une signification respectueuse de la Constitution, sans risquer de mettre en péril, voire d'annuler l'œuvre du législateur local.

C'est le même Tribunal fédéral qui a précisé que, pour certes l'obliger à appliquer en toute circonstance les lois votées par l'Assemblée fédérale, l'article 190 Cst. ne lui interdisait pas pour autant de constater, dans les considérants des arrêts qu'il rend, l'inconstitutionnalité d'une disposition législative. Pour certes leur faire obligation d'appliquer les lois fédérales, l'article 190 Cst. n'interdit pas pour autant aux juges fédéraux d'examiner la constitutionnalité de ces dernières. L'obligation que pose la disposition se limite en effet à l'application, en tout état, des lois fédérales, en sorte que le contrôle de leur conformité au droit supérieur, voire le constat d'une violation de ce dernier, n'est nullement proscrit.

Cette approche, dite de l' «Anwendungsgebot», par référence à la doctrine suisse alémanique, permet de réconcilier en quelque sorte la loi et la Constitution. Elle habilite en effet le Tribunal fédéral à examiner la compatibilité de la première à la seconde et à envoyer, au besoin, un message au Parlement fédéral afin que celui-ci prenne conscience de l'inconstitutionnalité qui affecte son œuvre et adopte les

\footnotetext{
${ }^{18}$ ATF 95 I 330 Jeckelmann. Dans sa pratique antérieure, le Tribunal fédéral refusait purement et simplement d'entrer en matière sur les recours mettant en cause la constitutionnalité de dispositions contenues dans des lois fédérales.
} 
mesures permettant de pallier cette carence ${ }^{19}$. Les cas ne sont plus rares, dans la jurisprudence récente, de lois fédérales ainsi passées au crible du contrôle de la constitutionnalité, étant précisé que, même déclarées inconstitutionnelles, les lois en cause doivent tout de même être appliquées ${ }^{20}$.

Enfin, c'est aussi le Tribunal fédéral qui, en proclamant le principe de la primauté du droit international sur le droit interne, a - au prix de certaines hésitations, il est vrai déclaré que le droit international, particulièrement les instruments de protection des droits de l'homme auxquels la Suisse est partie, priment en principe la législation interne, fût-elle contenue dans une loi fédérale.

Selon le Tribunal fédéral, la Constitution fédérale, en particulier en son article 190, n'instaure pas de rang hiérarchique entre les normes de droit international et celles de droit interne. Lorsqu'une contradiction insurmontable entre les deux ordres juridiques est constatée, la Haute Cour s'en tient à sa jurisprudence, selon laquelle le droit international public l'emporte en principe sur le droit interne, spécialement lorsque la norme internationale a pour objet la protection des droits de l'homme, mais également en dehors de toute question de protection des droits de l'homme, de sorte qu'une disposition légale de droit interne contraire ne saurait trouver application.

En d'autres termes, alors que l'article 190 Cst. fait obligation au Tribunal fédéral de faire primer la loi fédérale sur la Constitution, la même disposition fait primer un instrument de protection des droits de l'homme tel que la $\mathrm{CEDH}$ en cas de disposition contraire contenue dans la même loi fédérale. Comme les droits de l'homme garantis sur le plan conventionnel sont largement convergents avec leurs cousins tels qu'ils sont énoncés dans la Constitution fédérale au chapitre des droits fondamentaux, c'est bien une forme particulière et originale de juridiction constitutionnelle qui est ainsi exercée ${ }^{21}$.

La jurisprudence présente toutefois un tour fluctuant ${ }^{22}$. À l'occasion d'un arrêt récent, les juges fédéraux ont tenu à préciser que, face au refus de l'Assemblée fédérale de modifier la législation pour la rendre conforme aux canons du droit conventionnel, il s'estimait lié par ce choix de nature politique, faisant pour la circonstance prévaloir la norme interne contraire au droit européen ${ }^{23}$. II est vrai que ce précédent touche un

\footnotetext{
${ }^{19}$ Hansjörg Seiler, Verfassungsgerichtsbarkeit zwischen Verfassungsrecht, Richterrecht und Politik, Revue de droit suisse 2010 II, p. 401 et les références citées.

${ }^{20}$ ATF 117 Ib 367 Eidgenössische Steuerverwaltung ; 121 V 229 B. ; 123 II 9 C. ; 125 III 209 X.

${ }^{21}$ Hertig Randall (note 1), p. 249. Sur la question, voir AIJC XVIII-2002, p. 827 et AIJC XX-2004, p. 344 ss. et les références citées.

${ }^{22}$ Voir Hertig Randall (note 1), p. 251 ; Seiler (note 32), p. 403 et 465.

${ }^{23}$ Voir ATF 136 III 168 Ma., du 25 janvier 2010, consid. 3.3.4 : «Die verweigerte Änderung bzw. Anpassung des ehelichen Namensrechts an die EMRK bzw. aktuelle Rechtsprechung des Europäischen Gerichtshofs für Menschenrechte läuft darauf hinaus, dass der schweizerische Gesetzgeber bewusst den Grundsatz der Einheit der Familie und ihres Namens höher gewichtet als den Rechtsgleichheitsgrundsatz, wie ihn der Europäische Gerichtshof versteht. Damit liegt nahe, gemäss BGE 99 Ib 39 ff. i.S. Schubert das Bundesgesetz weiterhin als massgeblich zu erachten) (...), zumal eine Aufhebung der Pflicht zur gemeinsamen Namensführung sinnvollerweise mit der gesetzlichen Gestaltung des Namens der allfälligen gemeinsamen Kinder verbunden wird (...). Ob diese Sichtweise - Massgeblichkeit der geltenden ZGB-Bestimmungen - mit der neueren Rechtsprechung vereinbar ist, wonach das Bundesgericht der EMRK den Vorzug gibt, wenn eine Verurteilung durch den
} 
domaine particulièrement sensible, puisqu'il concerne la réglementation civile du nom de famille des conjoints, soit une problématique qui, suite à un arrêt de condamnation de la Suisse rendu par la Cour européenne des droits de l'homme en $1994^{24}$, n'a pas encore conduit le Parlement suisse à adapter une solution compatible avec la CEDH.

\subsubsection{Les modes de saisine de la juridiction constitutionnelle}

Les modes de saisine du Tribunal fédéral sont prévus par la LTF. Les voies de recours en matière civile, pénale et de droit public que prévoit cette loi fondent le mode quotidien de saisine de la Cour suprême. Comme la juridiction constitutionnelle s'exerce le plus souvent dans le cadre du contentieux ordinaire opposant des particuliers entre eux ou dans leurs rapports avec l'administration, l'Assemblée fédérale ne dispose d'aucune compétence pour intervenir dans ce cadre et déclencher elle-même le contentieux. Le contrôle préventif de constitutionnalité des lois fédérales reste inconnu en Suisse (Auer et al, 2006).

L'article 89 LTF pose les exigences qui entourent la qualité pour recourir devant le Tribunal fédéral. La disposition énumère, dans ce cadre, diverses instances susceptibles d'ester devant la Haute Cour. L'Assemblée fédérale n'y est pas mentionnée, excepté pour les litiges concernant les rapports de travail du personnel de la Confédération.

Le cas le plus fréquent d'exercice de la juridiction constitutionnelle oppose, devant le Tribunal fédéral, une ou plusieurs personnes physiques ou morales à un organe étatique, cantonal ou fédéral, comme indiqué précédemment. Les organes de l'Etat n'ont, de manière générale, guère l'occasion de déclencher eux-mêmes le contrôle de la constitutionnalité. En tant qu'il porte le plus souvent sur les droits fondamentaux protégés par la Constitution fédérale ou par la CEDH ou, plus rarement, par une constitution cantonale, ces droits sont dirigés contre les organes de l'Etat. Faute d'en être bénéficiaires, les détenteurs de la puissance publique ne sont en principe pas légitimés à invoquer leur violation.

II en va de même des lois cantonales. Le contrôle abstrait de constitutionnalité pratiqué à leur égard en application de l'article 82 lettre b LTF suppose que la saisine du Tribunal fédéral émane de particuliers, et non d'organes des cantons.

\subsubsection{Les modalités de délibération des cours constitutionnelles}

Comme indiqué précédemment, le contrôle de la répartition des compétences entre les différents pouvoirs de l'Etat fait partie des attributions que l'article 189 Cst. attribue au Tribunal fédéral. II s'agit d'un chapitre central, particulièrement complexe, de la juridiction constitutionnelle.

Europäischen Gerichtshof für Menschenrechte droht (...), braucht im konkreten Fall - wie sich aus dem Folgenden ergibt - nicht abschliessend entschieden zu werden. »

${ }^{24}$ ACEDH Burghartz c. Suisse du 22 février 1994, Série A, n 280-B. 
Les juges fédéraux sont habilités à juger que la répartition des compétences qu'effectue la Constitution fédérale entre la Confédération et les cantons est respectée. Le plus souvent, ce type de litige porte sur la compétence d'un canton pour légiférer dans un domaine déterminé. Le Tribunal fédéral doit alors interpréter la législation cantonale en cause et déterminer si la compétence incriminée revient effectivement au canton ou si elle ressortit, au contraire, à la Confédération. L'interprétation conforme à la Constitution joue un rôle déterminant dans ce contexte (Auer et al, 2006).

Ce n'est en effet que confronté à un conflit insoluble entre législation fédérale et cantonale que le Tribunal fédéral est appelé à trancher et, au besoin, à faire application de la règle de l'article 49 alinéa 1 Cst. selon laquelle le droit fédéral prime le droit cantonal qui lui est contraire. Ce type de contrôle s'exerce le plus souvent sur contrôle abstrait, voire concret des actes normatifs cantonaux, à la demande d'un particulier qui exerce un recours en matière de droit public au sens de l'article 82 LTF. Le Tribunal fédéral a par exemple été amené à juger que, bien que la compétence de réglementer la publicité pour l'alcool et le tabac revient à la Confédération, les cantons sont habilités à édicter des dispositions en ce domaine pour des raisons de politique sanitaire et de réglementation du domaine public ${ }^{25}$.

Le Tribunal fédéral peut aussi intervenir, mais c'est plus rare, lorsque, par la voie de l'action au sens de l'article 120 LTF, il est appelé à connaître en instance unique des conflits de compétence entre autorités, par exemple entre autorités cantonales. Ce type de contentieux conduit en pareille hypothèse les juges fédéraux à arbitrer des questions délicates relatives à la souveraineté des cantons. Dans un arrêt rendu le 25 octobre 1999, le Tribunal fédéral a par exemple jugé que les prétentions du fisc genevois d'imposer le revenu des travailleurs domiciliés dans le canton de Vaud, mais exerçant leur activité à Genève empiétait de manière indue sur la souveraineté fiscale vaudoise ${ }^{26}$.

Les conflits ouverts entre autorités fédérales et cantonales quant à l'exercice de leurs compétences respectives ne sont guère fréquents et prennent rarement une tournure judiciaire. Ils n'en sont pas moins fort intéressants. Un arrêt célèbre, rendu le 23 mars 1977 par le Tribunal fédéral ${ }^{27}$, a permis de fixer le cadre entourant l'installation de centrales nucléaires sur le plan cantonal. Dans cette affaire, qui portait sur l'installation d'une centrale nucléaire dans le canton de Genève, la Haute Cour a précisé qu'un désaccord entre la Confédération et un ou plusieurs cantons au sujet de l'étendue de leurs attributions relevait de son arbitrage en tant qu'il a pour objet la délimitation des souverainetés fédérale, d'une part, et cantonale, d'autre part. En l'espèce, les juges fédéraux ont considéré que la délivrance, par l'autorité fédérale compétente, de l'autorisation de site en faveur de l'implantation d'une centrale nucléaire ne permettait pas à l'entreprise bénéficiaire de construire cette installation au lieu choisi sans égard à la réglementation de zones instituée par le droit cantonal et communal. La division du territoire en différentes zones fait en effet partie des mesures d'aménagement qui relèvent de la compétence exclusive des cantons. Ces mesures appartiennent à la police des constructions au sens usuel du terme; elles

\footnotetext{
${ }^{25}$ ATF 128 I 295 Association suisse des annonceurs ; sur cet arrêt, voir AIJC XVIII-2002, p. 838 et les autres références citées.

${ }^{26}$ ATF 125 I 458 Canton de Vaud.

${ }^{27}$ ATF 103 Ia 329 Conseil d'Etat du canton de Genève.
} 
font partie des règles impératives de droit public limitant la faculté d'utiliser un immeuble et d'y construire. En déniant au canton de Genève le droit de procéder au déclassement des terrains destinés à la construction de la centrale nucléaire incriminée, l'autorité fédérale avait empiété sur les attributions du canton. Le Tribunal fédéral a donc admis la réclamation formée par le gouvernement genevois.

Le Tribunal fédéral bénéficie également de la compétence de connaître de recours formés pour violation de l'autonomie communale. Ce type de conflit porte sur la répartition des compétences entre un canton et l'une ou l'autre de ses communes. II s'agit d'un chef de compétence qui est expressément prévu par l'article 189 alinéa 1 lettre e Cst. et qui permet aux communes de défendre leur existence, leur territoire et, plus largement, les divers aspects qui composent leur autonomie ${ }^{28}$.

L'autonomie des communes est garantie à l'article 50 alinéa 1 Cst. II s'agit d'un droit fondamental qui permet de porter, en dernière instance, devant le Tribunal fédéral les litiges dans lesquels une commune ou un particulier se plaint de qu'une atteinte inadmissible au pouvoir municipal a été porté par une autorité cantonale. À travers le grief tiré de la violation de l'autonomie communale, le Tribunal fédéral peut statuer sur le respect des droits fondamentaux qui entretiennent un rapport étroit avec ce moyen comme la liberté économique, la garantie de la propriété, l'interdiction de l'arbitraire ou l'égalité de traitement.

Dans un arrêt du 8 décembre 2010, le Tribunal fédéral a par exemple jugé que le refus de la Ville de Genève de mettre à la disposition de l'artiste français Dieudonné une salle en vue de la présentation de son spectacle consacré à violence conjugale représentait une restriction disproportionnée de la liberté d'expression. L'autorité judiciaire cantonale, qui avait constaté cette violation de la part de la Ville de Genève, n'avait par conséquent pas porté atteinte à l'autonomie communale, Arrêt du Tribunal fédéral 1C_312/2010, Ville de Genève, Conseil administratif c. Djily Diagne, Pratique juridique actuelle 2011.

Le mode de fonctionnement des assemblées parlementaires ne fait pas, outre les cas évoqués ci-dessus, partie des compétences du Tribunal fédéral. En ce qui concerne l'activité de l'Assemblée fédérale, le recours au Tribunal fédéral n'est pas possible: l'article 189 alinéa 4 Cst. précise en effet que les actes qu'adopte cet organe, tout comme ceux du Conseil fédéral, ne peuvent pas être portés devant le Tribunal fédéral| ${ }^{29}$. Pour ce qui concerne l'activité des assemblées parlementaires cantonales, le contrôle abstrait des actes normatifs est possible au sens de l'article 82 lettre b LTF. Hormis ces chefs de compétence, les modalités d'activité et de fonctionnement de ces organes ne sont pas attaquables devant la Cour suprême.

\footnotetext{
${ }^{28}$ Sur le sujet, voir AIJC XXII-2006, p. 385 et les références citées.

${ }^{29}$ Seiler (note 32), p. 396.
} 


\section{LE CADRE SUBSTANTIEL DES RELATIONS ENTRE LES PARLEMENTS ET LES COURS CONSTITUTIONNELLES}

\subsection{L'espace occupé par le législateur dans la production normative et la concurrence avec d'autres acteurs}

La place qu'occupe le législateur au sens formel dans la production normative est centrale en Suisse. La loi en tant qu'ensemble de normes logiquement ordonnées, émanant d'une représentation fédéraliste et démocratique, constitue le point d'ancrage primaire de l'action de l'Etat, en tant qu'elle met en œuvre les mandats énumérés dans la Constitution. Le champ opératoire de la loi, sa distinction avec les autres types d'intervention de l'Etat et de ses modalités sont prévus de manière assez précise aux articles 163 et 164 Cst (Auer et al, 2006).

Pour être centrale, cette place n'est pas exclusive pour autant. La complexité de la vie contemporaine, alliée aux enjeux techniques sous-jacents à la plupart des enjeux législatifs et aussi au caractère milicien du Parlement, organe qui ne siège pas en permanence et qui n'est pas composé de professionnels de la politique, impliquent que la loi ne constitue le plus souvent qu'un point de départ. Une fois adoptées, les normes sont appelées à être précisées, interprétées et concrétisées. Ce processus de complètement et de concrétisation ne peut qu'émaner d'un organe autre, mieux outillé pour ainsi exécuter et, bien souvent, compléter le contenu des normes votées par le Parlement. Ce rôle revient au gouvernement qui, par voie d'ordonnances, précise les lois formelles. II en va de même sur le plan cantonal. À Genève par exemple, $80 \%$ environ de la production normative émanent du gouvernement cantonal.

Inévitablement, le juge constitutionnel peut être appelé à arbitrer les modalités qui entourent le phénomène de la délégation législative, c'est-à-dire le processus par lequel le législateur formel accepte de se défaire de certaines de ses compétences au profit du pouvoir exécutif ou même de l'administration, de même que l'ampleur du processus réglementaire. En opérant l'examen du rapport de l'ordonnance à la loi, le Tribunal fédéral est ainsi amené à assurer le respect de l'équilibre et la ligne de démarcation existant entre le législateur et l'exécutif. Ce contrôle s'exerce aussi bien à l'égard des ordonnances du Conseil fédéral qu'à l'égard des règlements édictés par le gouvernement des cantons.

Le développement progressif du droit international exerce lui aussi une influence considérable sur le travail législatif. Soit qu'ils remplacent purement et simplement la législation nationale, soit qu'ils fixent des limites que le législateur ne saurait franchir, les traités internationaux modèlent l'activité parlementaire.

Dans le domaine du droit des étrangers par exemple, le statut des ressortissants de I'Union européenne est régi depuis 2002 par l'Accord sur la libre circulation des personnes (ALCP), qui s'est substitué à la législation nationale ${ }^{30}$. S'il est certes exact que cet instrument a été approuvé par l'Assemblée fédérale puis soumis au vote populaire, il n'en demeure pas moins qu'il s'agit d'un instrument négocié à l'échelon

${ }^{30}$ Sur cet Accord, voir Auer/Malinverni/Hottelier (note 8), p. 142ss. ; AIJC XIX-2003, p. 851. 
international par le Conseil fédéral. Le juge constitutionnel ne peut qu'en prendre acte, en tirant au besoin les conclusions d'éventuels écarts entre la loi nationale et le droit international ${ }^{31}$.

Le Tribunal fédéral n'a pas manqué de se livrer à cet exercice dans un arrêt de principe rendu le 26 janvier $2010^{32}$. En l'espèce, la Haute Cour a jugé que le système d'imposition fiscale à la source pratiqué sur les revenus des travailleurs frontaliers employés dans le canton de Genève contrevenait à l'interdiction de discrimination contenue dans l'ALCP. Le système d'imposition variant, sous l'angle des déductions autorisées, entre frontaliers et travailleurs domiciliés à Genève, le Tribunal fédéral a constaté une discrimination au sens des articles 2 ALCP et 9 alinéa 2 de l'Annexe I à cet instrument.

Les juges fédéraux ont donné raison au recourant sur la base des dispositions précitées, en se référant abondamment à la jurisprudence développée par la Cour de justice des communautés européennes. De l'avis des juges fédéraux, la jurisprudence communautaire en matière de fiscalité directe constitue un cas d'application des règles générales d'égalité de traitement. Ces règles prohibent non seulement les discriminations ostensibles fondées sur la nationalité (discriminations directes), mais encore toutes les formes dissimulées de discrimination qui, par application d'autres critères de distinction, aboutissent en fait au même résultat (discriminations indirectes). À moins qu'elle ne soit objectivement justifiée et proportionnée à l'objectif poursuivi, une disposition de droit national doit, selon cette jurisprudence, être considérée comme indirectement discriminatoire dès lors qu'elle est susceptible, par sa nature même, d'affecter davantage les ressortissants d'autres Etats membres que les ressortissants nationaux et qu'elle risque, par conséquent, de défavoriser plus particulièrement les premiers. Si la fiscalité directe relève certes de la compétence des États membres, il n'en demeure pas moins que ces derniers doivent l'exercer dans le respect du droit communautaire. Ils sont tenus, par conséquent, de s'abstenir de toute discrimination ostensible ou déguisée fondée sur la nationalité.

Au final, le Tribunal fédéral a relevé que, alors même que d'après la règle de l'article 190 Cst., les juges fédéraux ne sont pas légitimés à refuser d'appliquer une loi fédérale, la jurisprudence qu'ils ont développée depuis une quinzaine d'années les a conduits à faire prévaloir le droit international public sur les lois fédérales dans le domaine des droits de l'homme comme dans les autres domaines ${ }^{33}$. En application de cette jurisprudence, l'ALCP et l'interdiction de discrimination l'emportent sur le droit interne contraire. L'instrument international déploie un effet direct en ce qui concerne, en particulier, le régime de l'imposition à la source des travailleurs frontaliers contenu dans la législation de rang fédéral. Le recours a ainsi été admis et la cause renvoyée aux instances cantonales afin que le recourant se voie appliquer, lors de son imposition à la source, le même régime de déductions fiscales que les contribuables résidant en Suisse qui sont soumis au régime d'imposition ordinaire.

\footnotetext{
${ }^{31}$ Sur le sujet, voir les développements de Hertig Randall (note 1), p. 264ss. et les références citées.

${ }^{32}$ ATF 136 II $241 X$. Sur cet arrêt, voir AIJC XXV-2009, p. 859.

${ }^{33}$ Sur le sujet, voir par exemple ATF 131 II 352 A. et ATF 133 V 367 Segretariato di Stato dell'economia.
} 
Les instruments internationaux de protection des droits de l'homme et, plus particulièrement la CEDH influencent notablement l'activité parlementaire, à l'échelon aussi bien fédéral que cantonal. L'éventualité d'une condamnation par la Cour européenne des droits de l'homme n'est évidemment pas étrangère à ce phénomène.

Le juge constitutionnel, lui-même à l'écoute des juges de Strasbourg, a également son mot à dire dans le processus législatif. Par les arrêts qu'il prononce, par l'interprétation de la Constitution à laquelle il procède, par l'ouverture au droit international dont il fait preuve dans le cadre du contrôle de conventionnalité, le Tribunal fédéral en vient souvent à énoncer des principes dont les parlementaires sont ensuite appelés à se faire l'écho.

\subsection{Les principaux axes d'évolution de la jurisprudence constitutionnelle}

\subsubsection{Les rapports entre les pouvoirs législatifs et les juridictions constitutionnelles}

En Suisse, les rapports entre pouvoir législatif et la juridiction constitutionnelle doivent être distingués selon que l'on parle du pouvoir législatif fédéral ou cantonal. S'agissant de l'Assemblée fédérale, la Constitution affirme l'idée de la prééminence de la loi formelle sur la sanction juridictionnelle fondée sur le respect de la Constitution. Comme indiqué précédemment, la règle de l'article 190 Cst. fait obligation au Tribunal fédéral d'appliquer les lois fédérales, que celles-ci respectent ou violent la Constitution. L'avènement de l'Etat de droit est ici éclipsé par la légitimité démocratique de la loi.

Le contrôle de constitutionnalité est en revanche possible, à titre incident, c'est-à-dire dans le cadre d'un contrôle concret, pour les autres actes normatifs fédéraux qui ne tombent pas sous le coup de l'article 190 Cst. II en va ainsi des ordonnances du Conseil fédéral, dans la mesure où elles ne reproduisent pas une inconstitutionnalité contenue dans la loi qui leur sert de fondement. Admettre l'inverse équivaudrait en effet à permettre, via le contrôle de l'ordonnance, à contrôler la constitutionnalité de la loi, d'une manière contraire à l'article 190 Cst.

La juridiction constitutionnelle est, en sens inverse, particulièrement développée à l'égard des lois cantonales. Ces dernières sont en effet soumises en dernier recours au contrôle du Tribunal fédéral, qui dispose du pouvoir d'annuler, dans le cadre du contrôle abstrait de constitutionnalité fondé sur l'article 82 lettre b LTF, celles qui contreviennent à la Constitution cantonale, à la Constitution fédérale, au droit international et au droit fédéral dans son ensemble. La logique, ici, réside dans la nécessité d'assurer une application uniforme du droit international et fédéral partout en Suisse et de permettre aux constitutions des cantons, qui bénéficient de la garantie de la Confédération que leur accorde l'Assemblée fédérale en application des articles 51 et 172 alinéa 2 Cst. Le contentieux concret et le contentieux abstrait se complètent de manière harmonieuse, tant il est vrai qu'ils procèdent d'un regard différente que pose le juge constitutionnel sur l'acte attaqué ${ }^{34}$.

\footnotetext{
${ }^{34}$ Sur le sujet, voir aussi les observations de Monsieur Jean-Claude Colliard, AIJC XXVI-2010, p. 17, à propos du Conseil constitutionnel français.
} 
L'argument démocratique ne fait par conséquent nullement écran au contrôle qu'exerce la Cour suprême de la Confédération face à une loi cantonale. De fait, même si cette dernière a été massivement acceptée lors d'un scrutin populaire cantonal, le Tribunal fédéral dispose du pouvoir de l'annuler si elle se révèle contraire à la Constitution. Un cas récent, mais déjà célèbre, permet d'illustrer le propos.

Un important arrêt rendu le $1^{\text {er }}$ juin $2007^{35}$ mettait en cause la constitutionnalité des barèmes fiscaux dégressifs applicables à l'impôt sur le revenu et la fortune qui avaient récemment été adoptés le canton d'Obwald. Progressifs pour les premières tranches de revenu et de fortune imposables dans le canton, les barèmes affichaient une tendance dégressive à partir de 300'000.- francs de revenu annuel et 5 millions de francs de fortune. Soumise à référendum sur le plan local, cette loi avait été plébiscitée par près de $80 \%$ de suffrages favorables. Saisi d'un recours exercé par plusieurs citoyens, le Tribunal fédéral a néanmoins déclaré ce système fiscal contraire aux principes de l'égalité et de l'imposition en fonction de la capacité économique du contribuable.

87. Dans le cadre du débat, assurément passionnant, portant sur les rapports entre la démocratie directe, le respect de la structure fédérale en matière de finances publiques et celui des droits fondamentaux, la Haute Cour a ajouté, à l'issue d'un examen particulièrement approfondi, que ni des motifs de concurrence fiscale entre cantons, ni d'autres buts - qu'ils fussent de nature fiscale ou non - ne permettaient de remédier à l'atteinte aux principes constitutionnels précités ${ }^{36}$.

2.2.2. La place de la juridiction constitutionnelle dans le débat politique et dans le débat législatif

88. La place qu'occupe la juridiction constitutionnelle dans le débat politique est centrale. À l'égard des actes normatifs de rang cantonal, cette situation s'explique par le fait qu'institutionnellement, le Tribunal fédéral bénéficie de compétences étendues. Celles-ci ne lui permettent pas seulement de contrôler la conformité à la Constitution fédérale de toute décision fondée sur le droit cantonal ou communal, mais aussi, dans le cadre du contrôle abstrait, d'annuler toute norme de rang législatif ou réglementaire local.

Ce pouvoir de contrôle exerce, en amont, une influence non négligeable sur le mode de production des actes normatifs cantonaux. Bien souvent, le législateur cantonal s'efforce d'adopter des textes en s'entourant de conseils et d'avis sur leur conformité au droit supérieur. En dernier recours, c'est bien le Tribunal fédéral qui peut exercer le contrôle de constitutionnalité et de conventionnalité.

Le Tribunal fédéral, conformément aux articles 189 alinéa 4 et 190 Cst., ne peut pas annuler une loi fédérale, pas plus du reste que les ordonnance qu'édicte le Conseil fédéral. Lorsqu'il est possible, le contrôle de constitutionnalité ne porte que sur des décisions d'application de normes fédérale. II ne s'agit que d'un contrôle concret. L'influence du Tribunal fédéral ne saurait être sous-estimée pour autant.

\footnotetext{
${ }^{35}$ ATF 133 I 206 Halter-Durrer und Mitb. ; voir AIJC XXIII-2007, p. 931.

${ }^{36}$ Sur la question, voir également AIJC XXII-2006, p. 509ss.
} 
Lorsqu'il se prononce sur la validité d'une décision fédérale non immunisée par la règle de l'article 190 Cst., le Tribunal fédéral dispose en effet du pouvoir d'annuler l'acte concrètement attaqué. Si la décision en cause ne fait qu'appliquer le contenu de l'acte normatif, c'est bien ce dernier qui, indirectement, est déclaré contraire à la Constitution. À charge ensuite pour son auteur - le Conseil fédéral - de le reprendre et de le modifier afin de le rendre conforme au droit supérieur.

Un cas original permet d'illustrer l'importance de ce qui précède. Durant les années 1970, après avoir adopté une ordonnance obligeant à équiper les véhicules immatriculés en Suisse d'être dotés de ceintures de sécurité, le Conseil fédéral prit la décision d'en rendre le port obligatoire. II adopta donc une ordonnance en ce sens en date du 10 mars 1975 , laquelle entra en vigueur le $1^{\mathrm{er}}$ janvier $1976^{37}$. En mars 1976, un automobiliste valaisan, qui n'avait pas attaché la ceinture, fut amendé. L'intéressé porta le litige jusque devant le Tribunal fédéral et souleva deux arguments. Selon le premier, l'ordonnance du Conseil fédéral n'avait pas de base légale, faute de reposer sur une norme de délégation législative votée à cet effet par l'Assemblée fédérale. Selon le second, être obligé de boucler sa ceinture en voiture contrevenait à la liberté personnelle protégée par la Constitution fédérale.

Dans un arrêt du 2 septembre 1977, le Tribunal fédéral admit le premier moyen ${ }^{38}$. Aucune norme législative adoptée par l'Assemblée fédérale, soumise par conséquent au débat démocratique, voire au référendum populaire, n'avait délégué au Conseil fédéral la compétence de rendre obligatoire le port de la ceinture de sécurité pour les automobilistes. L'organe gouvernemental avait donc outrepassé ses compétences. L'amende - en l'espèce, 20 francs... - fut annulée, les juges fédéraux ayant constaté qu'elle mettait en cause une obligation de faire dépourvue de toute base légale. L'argument tiré de la violation de la liberté personnelle fut laissé indécis au nom de l'économie de procédure. Mais dans un autre arrêt, rendu le 5 octobre 1977 et mettant en cause un automobiliste neuchâtelois, le Tribunal fédéral considéra que le port de la ceinture de sécurité, pour constituer une restriction à la liberté personnelle, devait reposer sur une base légale. Celle-ci faisant précisément défaut, la Haute Cour admit également ce recours et annula l'amende litigieuse, sans examiner si la restriction était justifiée ${ }^{39}$.

La conséquence de cette affaire, passablement médiatisée à l'époque fut que, suite à l'arrêt du Tribunal fédéral, l'ordonnance du Conseil fédéral prévoyant le port de la ceinture de sécurité ne fut plus appliquée dans aucun canton. Un projet de loi fut déposé par le Conseil fédéral ${ }^{40}$. Modifiant la loi fédérale sur la circulation routière, il permettait aux Chambres de déléguer au Conseil fédéral la compétence de rendre obligatoire le port de la ceinture de sécurité aux automobilistes. La loi ainsi modifiée fut votée le 21 mars $1980^{41}$. Frappée d'une demande de référendum formée par plus de 50'000 citoyens, elle fut soumise au scrutin populaire. Le 30 novembre 1980, la

\footnotetext{
${ }^{37}$ Voir Jean-François Aubert, Exposé des institutions politiques de la Suisse à partir de quelques affaires controversées, $2^{\text {ème }}$ éd., Lausanne 1983, p. 190ss.

${ }^{38}$ ATF 103 IV 192 Favre.

${ }^{39}$ Aubert (note 54), p. 194. Curieusement, cet arrêt n'a pas fait l'objet de publication.

${ }^{40}$ Voir Aubert (note 54), p. 193 : « Le contrôle judiciaire conduit ainsi au contrôle politique. »

${ }^{41}$ Il s'agit de l'article 57 alinéa 5 de la loi fédérale sur la circulation routière, du 19 décembre 1958 ; RS 741.01.
} 
novelle a été acceptée par le peuple. Suite à cette acceptation, le Conseil fédéral a adopté une nouvelle ordonnance rendant obligatoire le port de la ceinture de sécurité en voiture, de même que le port du casque pour les motocyclistes. Ce régime, toujours en vigueur, est applicable depuis le $1^{\text {er }}$ juillet $1981^{42}$.

Dans un sens assez proche, le pouvoir du Tribunal fédéral de constater qu'une norme contenue dans une loi fédérale contrevient à la Constitution permet d'attirer l'attention des parlementaires sur la nécessité de procéder à une réforme législative. Ce pouvoir n'est certes qu'indicatif ou informatif, l'article 190 Cst. faisant en tout état obligation aux juges d'appliquer les lois fédérales. II s'agit cependant d'un signal institutionnel important, qui est susceptible de conduire à la réforme de normes législatives dans le souci de les rendre compatibles avec la Constitution.

Enfin, le développement du contrôle de la conventionnalité permet au Tribunal fédéral, au nom du respect du droit international, de refuser d'appliquer les normes législatives contraires par exemple à la CEDH. À la suite d'un arrêt de la Cour européenne des droits de l'homme sanctionnant la Suisse pour violation de la présomption d'innocence dans le cas d'héritiers condamnés pour des infractions à la législation fiscale commises, à l'époque, par le de cuius $^{43}$, le Tribunal fédéral a précisé qu'il refuserait d'appliquer, à l'avenir, la disposition litigieuse ${ }^{44}$. L'Assemblée fédérale a par la suite abrogé la disposition litigieuse.

Dans un sens comparable, le législateur fédéral a abrogé la disposition du Code civil qui habilitait le juge du divorce à fixer au conjoint responsable du divorce un délai pendant lequel il était privé du droit de se remarier, suite à l'arrêt par lequel la Cour de Strasbourg avait condamné la Suisse pour violation de l'article $12 \mathrm{CEDH}^{45}$.

\subsubsection{L'intégration des exigences constitutionnelles dans les débats parlementaires}

Lorsqu'elle élabore et vote une loi, l'Assemblée fédérale est censée respecter la Constitution. Tout message du Conseil fédéral présenté à l'appui d'un projet de loi est accompagné d'un chapitre examinant la question de la conformité à la Constitution. Celui-ci indique les bases légales ou constitutionnelles sur lesquelles le projet se fonde, ses effets sur les droits fondamentaux, sa compatibilité avec le droit de rang supérieur et ses relations avec le droit européen (art. 141 al. 2 let. a LParl.). La norme qui fonde son caractère d'autorité suprême de la Confédération réserve en effet, comme indiqué précédemment, le respect des droits du peuple et des cantons, c'est-à-dire les droits de l'organe constituant, donc de la Constitution.

Cette proclamation de principe ne confère toutefois pas l'assurance d'un respect systématique des normes constitutionnelles, ce d'autant que l'absence d'une

\footnotetext{
${ }^{42}$ Il s'agit de l'article $3 a$ de l'ordonnance du Conseil fédéral sur les règles de la circulation routière, du 13 novembre 1962 ; RS 741.11. Sur l'historique des travaux de l'Assemblée fédérale, voir Aubert (note 54), p. 331.

${ }^{43}$ ACEDH A.P., M.P. et T.P. c. Suisse, arrêt du 29 août 1997, Rec. 1997-V, p. 1477 ; E.L., R.L. et J.O.-L. c. Suisse, arrêt du 29 août 1997, Rec. 1997-V, p. 1509.

${ }^{44}$ ATF 124 II 480 Erben $P$.

${ }^{45}$ ACEDH F. c. Suisse, arrêt du 18 décembre 1987, Série A, n 128 . Voir Michel Hottelier/Hanspeter Mock/Michel Puéchavy, La Suisse devant la Cour européenne des droits de l'homme, $2^{\text {ème }}$ éd., Zurich 2011, p. 27 et les références citées.
} 
véritable juridiction constitutionnelle à l'égard des lois fédérales telle qu'elle découle de l'article 190 Cst. limite effectivement le pouvoir d'intervention des juges fédéraux. Cela étant, lorsque le constat d'une violation de la Constitution par une loi fédérale est posé, cet élément ne manque pas d'investir le débat parlementaire.

2.2.4. La gestion des conflits entre le créateur de la norme législative et la juridiction constitutionnelle

La gestion des conflits entre l'auteur de la norme législative et la juridiction constitutionnelle dépend des situations. Lorsque la norme législative est d'essence cantonale, le dernier mot revient au Tribunal fédéral si celui-ci constate que le droit supérieur n'est pas respecté. La primauté du droit fédéral sur le droit cantonal contraire déploie ici un effet marqué.

Lorsque la norme législative est d'essence fédérale, la situation se présente sous un jour plus complexe. Si la Constitution n'est pas respectée par le législateur, celui-ci peut être invité à élaborer une norme conforme. L'arrêt du Tribunal fédéral se limite toutefois, au plus, à un constat, sans effet cassatoire ni sur la décision attaquée, ni sur l'acte parlementaire qui lui sert de fondement. Et si, en dépit de ce constat, le législateur fédéral ne modifie pas la loi, le Tribunal fédéral s'estime lié.

Ce cadre se transforme dans l'hypothèse d'un contrôle de conventionnalité. Dans ce cas, au nom du membre de phrase de l'article 190 Cst. qui lui fait obligation d'appliquer le droit international, le Tribunal fédéral peut en arriver à refuser d'appliquer la norme fédérale. La jurisprudence récente tempère toutefois cette issue institutionnelle. Dans l'hypothèse où, de manière délibérée, le Parlement fédéral refuse ou ne parvient pas à conférer à la norme une tournure conforme au droit international, le Tribunal fédéral s'estime lié par cette solution. II applique donc la norme, sans égard à son inconventionnalité. L'hypothèse d'un constat, par la Cour européenne des droits de l'homme, d'un manquement de la Suisse à ses engagements internationaux demeure alors, bien entendu, réservé ${ }^{46}$.

\section{CONCLUSION}

L'exposé qui précède démontre que les rapports entre juges constitutionnels et parlements ne se laissent pas aisément appréhender en Suisse. Si la souveraineté, au sens historique du terme, occupe une place centrale dans cette problématique, on aurait tort de croire qu'elle l'épuise. En réalité, l'Etat moderne présente un visage sensiblement plus complexe, qui dépasse et transcende assez largement l'opposition traditionnelle entre parlementarisme et démocratie directe d'une part, compétence du juge constitutionnel de l'autre.

Le système suisse de justice constitutionnelle, certes assez sophistiqué, a subi une évolution remarquable au cours du vingtième siècle. Sans conteste, l'avis des magistrats constitutionnels s'invite de plus en plus dans le débat parlementaire et, plus largement, dans le débat politique. L'influence croissante du droit international, en particulier depuis le dernier quart du siècle dernier, tout particulièrement dans le

\footnotetext{
${ }^{46}$ ATF 136 III 168 Ma.
} 
domaine des droits de l'homme, révèle que l'Etat contemporain ne tire pas sa légitimité du scrutin populaire.

L'Etat démocratique est aussi un Etat de droit, au nom précisément du respect inconditionnel des principes cardinaux de respect de la dignité humaine, de protection des droits les plus élémentaires de la personne et de la protection des minorités. Pour répondre à la question posée en titre du présent rapport, le conflit historique de légitimité a progressivement cédé, à notre sens du moins, la place à une nouvelle forme d'organisation des pouvoirs.

Cette vision d'un Etat fondé non pas sur l'antagonisme, mais bien la complémentarité entre démocratie, fédéralisme et droits de la personne humaine, a conduit à une réinterprétation fondamentale de l'article 190 Cst., qui fait historiquement interdiction au Tribunal fédéral de contrôler la constitutionnalité des lois fédérales.

Cette disposition voit, de nos jours, son champ opératoire de plus en plus réduit à la seule obligation d'appliquer les textes de l'Assemblée fédérale contraires à la Constitution, alors même que les juges s'estiment légitimés à écarter ces mêmes lois lorsqu'elles contreviennent au droit international. Le processus n'est toutefois pas encore achevé, en raison de la persistance, dans la Constitution fédérale, de l'obligation inconditionnelle d'appliquer les lois fédérales, qu'elles soient conformes ou contraires à la Constitution fédérale, figurant à l'article 190 Cst.

Reste à savoir combien de temps encore, en particulier au prix de combien de condamnations de la Suisse par la Cour européenne des droits de l'homme, le système suisse de justice constitutionnelle pourra continuer à afficher deux types de contrôle fondés sur une approche du respect du droit supérieur aussi contradictoire.

\section{RÉFÉRENCES BILIOGRAFIQUES}

- ACEDH, Burghartz C. (1994). Série A, n²80-B. Suisse.

- Aubert J. et Mahon P. (2003). Petit commentaire de la Constitution fédérale de la Confédération suisse du 18 avril 1999. Zurich.

- Aubert J. (1967), Traité de droit constitutionnel suisse. Neuchâtel.

- Aubert J. (1967). Traité de droit constitutionnel suisse. Neuchâtel.

- Aubert J.-F. (1983). Exposé des institutions politiques de la Suisse à partir de quelques affaires controversées (2ème éd.). Lausanne.

- Auer A., Malinverni G., et Hottelier M. (2006). Droit constitutionnel suisse (vol. I, $2^{\text {ème }}$ éd.), Berne.

- Hertig M. (2010). L'internationalisation de la juridiction constitutionnelle : défis et perspectives, Revue de droit suisse, volume II, p. 230.

- Hottelier M., Mock H., et Puéchavy M.( 2011). La Suisse devant la Cour européenne des droits de l'homme (2ème éd.), Zurich.

- Kiener R. (2001). Richterliche Unabhängigkeit. Berne.

- Poltier E. (2011). L'organisation et le fonctionnement interne de l'ordre judiciaire et des tribunaux, Pratique juridique actuelle. 
- Seiler H. (2010). Verfassungsgerichtsbarkeit zwischen Verfassungsrecht, Richterrecht und Politik, Revue de droit suisse, volume II, p. 401.

- Suisse, Constitution fédérale de la Confédération suisse du 18 avril 1999 (2003), Zurich.

- Suisse, Cour Constitutionnelle (1997, août), « arrêt Rec. 1997-V », Berna.

- Suisse, Cour Constitutionnelle (1997, août), « arrêt Rec. 1997-V », Berna. 